\title{
Dimethylenastron suppresses human pancreatic cancer cell migration and invasion in vitro via allosteric inhibition of mitotic kinesin Eg5
}

\author{
Xiao-dong SUN ${ }^{1, \#}$, Xing-juan SHI ${ }^{1, \#}$, Xiao-ou SUN ${ }^{1, \#}$, You-guang LUO ${ }^{1}$, Xiao-jing WU ${ }^{1}$, Chang-fu YAO ${ }^{1}$, Hai-yang YU ${ }^{1}$, \\ Deng-wen $\mathrm{LI}^{1}$, Min $\mathrm{LIU}^{2, *}$, Jun $\mathrm{ZHOU}^{1, *}$ \\ ${ }^{1}$ Department of Genetics and Cell Biology, Tianjin Key Laboratory of Protein Science, College of Life Sciences, Nankai University, Tianjin \\ 300071, China; '2Department of Biochemistry, Key Laboratory of Immune Microenvironment and Disease of the Ministry of Education, \\ Basic Medical College, Tianjin Medical University, Tianjin 300070, China
}

Aim: The mitotic kinesin Eg5 plays a critical role in bipolar spindle assembly, and its inhibitors have shown impressive anticancer activity in preclinical studies. This study was undertaken to investigate the effect of dimethylenastron, a specific inhibitor of Eg5, on the migration and invasion of pancreatic cancer cells.

Methods: Human pancreatic cancer cell lines PANC1, EPP85, BxPC3, CFPAC1, and AsPAC1 were used. Eg5 expression was examined using immunofluorescence microscopy. Cell migration and invasion were analyzed with wound healing and transwell assays. Cell proliferation was examined using sulforhodamine B and MTT assays. The binding of dimethylenastron to Eg5 was analyzed with a molecular modeling study, and the ADP release rate was examined with the MANT-ADP reagent.

Results: Eg5 expression was 9-16-fold up-regulated in the 5 pancreatic cancer cell lines. Treatment of PANC1 pancreatic cancer cells with dimethylenastron ( 3 and $10 \mu \mathrm{mol} / \mathrm{L}$ ) for $24 \mathrm{~h}$ suppressed the migratory ability of the cancer cells in a concentration-dependent manner. The invasion ability of the cancer cells was also reduced by the treatment. However, treatment of PANC1 cells with dimethylenastron ( 3 and $10 \mu \mathrm{mol} / \mathrm{L}$ ) for $24 \mathrm{~h}$ had no detectable effect on their proliferation, which was inhibited when the cancer cells were treated with the drug for $72 \mathrm{~h}$. Molecular modeling study showed that dimethylenastron could allosterically inhibit the motor domain ATPase of Eg5 by decreasing the rate of ADP release.

Conclusion: Dimethylenastron inhibits the migration and invasion of PANC1 pancreatic cancer cells, independent of suppressing the cell proliferation. The findings provide a novel insight into the mechanisms of targeting Eg5 for pancreatic cancer chemotherapy.

Keywords: kinesin; Eg5; dimethylenastron; pancreatic cancer; cell migration; cell invasion; cell proliferation; molecular modeling

Acta Pharmacologica Sinica(2011) 32: 1543-1548; doi: 10.1038/aps.2011.130; published online 10 Oct 2011

\section{Introduction}

Eg5 (also known as kinesin-5, kinesin spindle protein, or Kif11) is encoded by the KIF-11 gene located at chromosome $10 \mathrm{q} 24.1$. As a member of the BimC family of kinesin-related proteins, Eg5 is a microtubule-dependent motor protein and plays a crucial role in the assembly and maintenance of the bipolar spindle, by hydrolysis of ATP to generate outward forces and push apart anti-parallel microtubules ${ }^{[1,2]}$. In addition, accumulating evidence indicates that Eg5 is highly expressed in cancer cell lines and tumor samples ${ }^{[3,4]}$. It has been reported that the

\footnotetext{
\#These authors contributed equally to this work.

* To whom correspondence should be addressed.

E-mail minliu@tijmu.edu.cn (Min LIU); junzhou@nankai.edu.cn (Jun ZHOU)

Received 2011-07-13 Accepted 2011-09-06
}

overexpression of Eg5 leads to abnormal spindle formation, genomic instability, and the development of a broad spectrum of cancers ${ }^{[3,4]}$.

Eg5 has been demonstrated as an effective target for cancer treatment. Antisense oligonucleotides against Eg5 has been shown to reduce the growth of tumors in xenograft models ${ }^{[5]}$. In blast crisis chronic myeloid leukemia and prostate cancer cells, in which Eg5 is highly expressed, inhibition of Eg5 causes cell cycle arrest and significantly suppresses cell proliferation $^{[5,6]}$. Over the last decade, the effects of various Eg5 inhibitors on the proliferation of cancer cells have been investigated, and the mechanisms of action of several Eg5 inhibitors have been studied ${ }^{[7-11]}$. Dimethylenastron is a cell-permeable quinazoline-thione compound that acts as a potent inhibitor of $\mathrm{Eg}^{[12]}$. We have demonstrated previously that dimethylenastron inhibits pancreatic tumor growth by suppressing cell 
proliferation and resulting in robust apoptosis ${ }^{[11]}$.

Pancreatic cancer is a highly malignant neoplasm of the pancreas and the fourth leading cause of cancer-related deaths worldwide. The prognosis of this disease is poor, with fewer than $5 \%$ of those diagnosed still alive five years after diagnosis ${ }^{[13]}$. The high mortality rate of pancreatic cancer results mainly from the delay in diagnosis and the high rate of metastasis, of which abnormal cancer cell motility is an essential component ${ }^{[14,15]}$. It remains elusive whether Eg5 inhibitors affect cancer cell motility, despite the intensive studies of the mechanisms of action of this group of compounds. In this study, we provide the first evidence that dimethylenastron allosterically inhibits Eg5 activity and reduces the migration and invasion of pancreatic cancer cells, independent of its inhibitory effect on pancreatic cancer cell proliferation.

\section{Materials and methods Materials}

Dimethylenastron was purchased from Calbiochem. Sulforhodamine B, 3-(4,5-dimethyl-2-thiazolyl)-2,5-diphenyl-2H-tetrazolium bromide (MTT), and 4'-6-diamidino-2-phenylindole (DAPI) were from Sigma-Aldrich, and the mouse monoclonal antibody against Eg5 was from Abcam. The rhodamineconjugated anti-mouse secondary antibody was obtained from Amersham Biosciences.

\section{Cell culture}

PANC1, EPP85, BxPC3, CFPAC1, and AsPC1 human pancreatic cancer cells were cultured in RPMI-1640 medium supplemented with $2 \mathrm{mmol} / \mathrm{L}$ L-glutamine and 10\% fetal bovine serum at $37^{\circ} \mathrm{C}$ in a humidified atmosphere with $5 \% \mathrm{CO}_{2}$. Normal pancreatic epithelial cells were grown as primary cultures as described previously ${ }^{[16]}$.

\section{In vitro cell proliferation assay}

Cells grown in 96-well plates were treated with gradient concentrations of dimethylenastron for 24 or $72 \mathrm{~h}$. Sulforhodamine B and MTT assays were then performed as described previously ${ }^{[17]}$. The percentage of cell proliferation as a function of drug concentration was plotted.

\section{Immunofluorescence microscopy}

Cells grown on glass coverslips were fixed with cold $\left(-20^{\circ} \mathrm{C}\right)$ methanol for $8 \mathrm{~min}$ and then washed with phosphate-buffered saline (PBS). Nonspecific sites were blocked by incubating with $2 \%$ bovine serum albumin diluted with PBS for $20 \mathrm{~min}$ at room temperature. Cells were incubated with mouse monoclonal anti-Eg5 antibody (1:100 dilution) for $2 \mathrm{~h}$ and then with rhodamine-conjugated anti-mouse secondary antibody for $2 \mathrm{~h}$, followed by staining with DAPI for $5 \mathrm{~min}$. Coverslips were mounted with $90 \%$ glycerol in PBS and examined with a Zeiss fluorescence microscope. Images of interphase cells were taken, and 300 cells were analyzed. Eg5 expression in cells was quantified by measuring the fluorescence intensity of Eg5 with AxioVision Rel 4.1 software. To quantify Eg5 distribution in the nucleus, an irregular circle was drawn outside the nucleus, and the fluorescence intensity of Eg5 within this circle was measured. The percentage of Eg5 expression in the nucleus was then calculated.

\section{Wound healing assay}

Cells grown in serum-free medium for $12 \mathrm{~h}$ as confluent monolayers were treated with dimethylenastron and mechanically scratched with a $20 \mu \mathrm{L}$ pipette tip to create the wound. Cells were then washed with PBS to remove the debris, and culture medium containing $10 \%$ or $5 \%$ serum was added to allow for wound healing. Phase-contrast images of the wound were taken $24 \mathrm{~h}$ later, and the number of cells that migrated into the wound area was counted in three random fields per wound. The extent of wound closure was quantified as the number of migrating cells in the drug-treatment group divided by the number of migrating cells in the control group.

\section{Transwell invasion assay}

Cell invasion in response to dimethylenastron was carried out by transwell assays. The upper surface of the transwell filters was coated with matrigel or fibronectin. Cells suspended in $200 \mu \mathrm{L}$ serum-free media were added to the chamber, and the chamber was placed in a 24 -well plate containing complete medium. After $24 \mathrm{~h}$ of incubation at $37^{\circ} \mathrm{C}$, the filters were gently taken out and matrigel on the upper surface of the filters was removed by cotton swabs. Cells on the underside of transwell filters were fixed with $4 \%$ paraformaldehyde for 30 min, stained with $0.1 \%$ crystal violet for $10 \mathrm{~min}$, and then photographed. For quantitative assessment, the number of invading cells was counted in five random fields per filter. The extent of cell invasion was quantified as the number of invading cells in the drug-treatment group divided by the number of invading cells in the control group.

\section{Molecular modeling}

Dimethylenastron was docked onto the coordinates obtained from the crystal structure of $\mathrm{Eg} 5^{[18]}$, using standard DOCK methodology ${ }^{[19]}$. The lowest-energy Eg5/dimethylenastron interaction model was presented.

\section{Measurement of ADP release rate}

The effects of dimethylenastron on the ADP release rate of the Eg5 motor domain were examined with the MANTADP reagent (Molecular Probes), by measuring the changes in MANT-ADP fluorescence as described previously ${ }^{[9,20]}$. In brief, purified Eg5 motor domain was incubated with the MANT-ADP racemate at a 1:1 stoichiometry. The Eg5-MANTADP complex was then mixed with $1 \mathrm{mmol} / \mathrm{L} \mathrm{MgATP}$ in the presence or absence of preformed microtubules. The decrease in MANT-ADP fluorescence was then examined over time at an excitation wavelength of $360 \mathrm{~nm}$ and an emission wavelength of $460 \mathrm{~nm}$. The observed exponential rate constant was then fit to a hyperbola to obtain the ADP release rate.

\section{Preparation of microtubules}

For the preparation of microtubules, $10 \mu \mathrm{mol} / \mathrm{L}$ microtubule 
associated protein-free tubulin (cytoskeleton) was incubated at $35^{\circ} \mathrm{C}$ for $30 \mathrm{~min}$ in a buffer containing $80 \mathrm{mmol} / \mathrm{L} \mathrm{Na}$-PIPES (pH 6.9), $1 \mathrm{mmol} / \mathrm{L} \mathrm{MgCl}_{2}, 1 \mathrm{mmol} / \mathrm{L}$ EGTA, $1 \mathrm{mmol} / \mathrm{L}$ GTP, and $5 \%$ glycerol.

\section{Statistical analysis}

Student's $t$ test was used to determine statistical significance. $P$ values less than 0.05 were considered significant, and $P$ values less than 0.01 were considered highly significant. Experiments were repeated 3 times, and error bars represent standard deviations between experiments.

\section{Results}

Eg5 expression is up-regulated in pancreatic cancer cell lines

To investigate the potential of Eg5 as a target for pancreatic cancer treatment, we examined its expression in several well characterized pancreatic cancer cell lines, including BxPC3, PANC1, EPP85, CFPAC1, and AsPAC1, and compared to Eg5 expression in normal pancreatic epithelial cells. Consistent with previous studies ${ }^{[4]}$, we found that Eg5 was highly expressed in all the pancreatic cancer cell lines examined (Figure 1A-1B). Interestingly, while only a small portion of Eg5 was localized in the nucleus in normal cells, a significant increase in its nuclear localization was observed in pancreatic cancer cells (Figure 1C).

\section{Inhibition of Eg5 activity suppresses pancreatic cancer cell migration}

The overexpression of Eg5 in pancreatic cancer cells prompted us to examine whether the inhibition of Eg5 activity affects the motility of pancreatic cancer cells. PANC1 cells were used for the analysis of cell motility because of their well-characterized genetic background, growth conditions, and migratory abil${ }^{i t y}{ }^{[21]}$. Serum-starved PANC1 cells grown as confluent monolayers were treated with the Eg5 inhibitor dimethylenastron and mechanically scratched to create the wound. Culture medium containing $10 \%$ serum was then added to allow wound healing, and phase-contrast images of the wound were taken $24 \mathrm{~h}$ later to examine the extent of wound closure as a measure of cell migration. As shown in Figure 2A-2B, dimethylenastron inhibited pancreatic cancer cell migration in a concentration-dependent manner, as evidenced by compromised wound closure in response to dimethylenastron treatment. The inhibitory effect of dimethylenastron on cell migration was more evident when culture medium containing $5 \%$ serum was used for wound healing (Figure 2C).

\section{Dimethylenastron inhibits pancreatic cancer cell invasion}

We then performed transwell assays to evaluate the effect of inhibition of Eg5 activity on pancreatic cancer cell invasion. PANC1 cells were treated with dimethylenastron and loaded to the transwell chambers (the upper surface of the transwell filters was coated with matrigel). After $24 \mathrm{~h}$, cells migrated to the underside of the transwell filters were stained with crystal violet solution and imaged. As shown in Figure 3A-3B, inhibition of Eg5 activity by dimethylenastron significantly
A
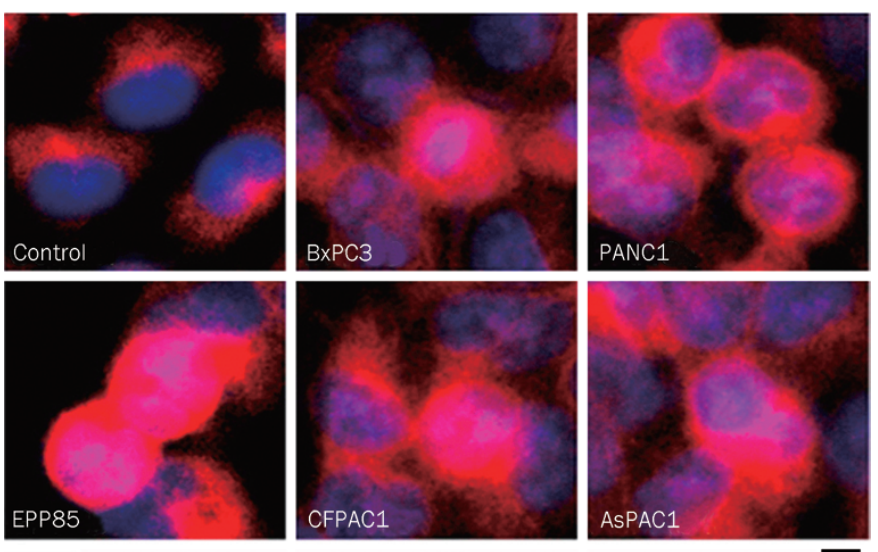

B

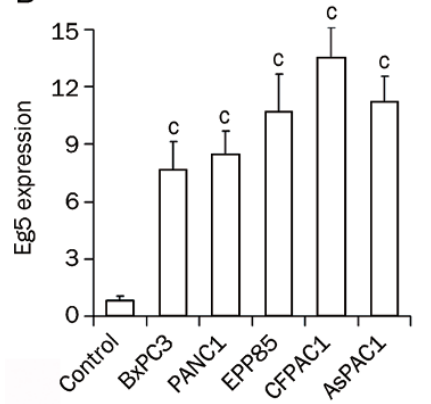

C

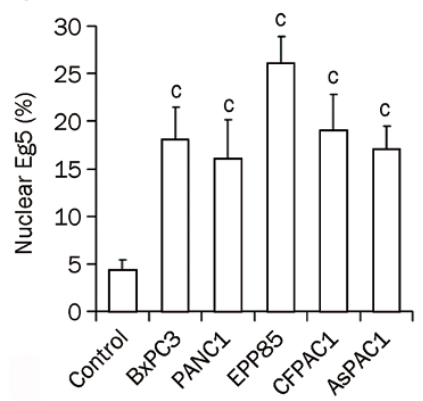

Figure 1. Overexpression of Eg5 in pancreatic cancer cell lines. (A) Immunofluorescence microscopic analysis of Eg5 (red) in pancreatic cancer cells and control normal pancreatic epithelial cells. Cells were also stained with the DNA dye DAPI to show the nuclei (blue). All the cells shown in this figure are interphase cells. Scale bar, $10 \mu \mathrm{m}$. (B) Experiments were performed as in $A$, and the expression of Eg5 in cells was quantified. (C) Experiments were performed as in A, and the distribution of Eg5 in the nucleus was quantified. Mean \pm SD. $n=3$. ${ }^{\mathrm{c}} P<0.01$ vs control.

suppressed the ability of pancreatic cancer cells to invade to the underside of the transwell filters. Similar results were achieved when the upper surface of the transwell filters was coated with fibronectin (Figure 3C).

\section{Dimethylenastron inhibits pancreatic cancer cell proliferation in} a concentration-dependent manner

We then examined whether the reduced migration and invasion of pancreatic cancer cells in response to dimethylenastron results from its effect on cell proliferation. To investigate this possibility, sulforhodamine B and MTT-based cell proliferation assays were performed. As shown in Figure 4A, both sulforhodamine B and MTT assays revealed that at concentrations 1 to $10 \mu \mathrm{mol} / \mathrm{L}$, dimethylenastron did not obviously affect PANC1 cell proliferation when cells were treated for 24 h. In contrast, 1 to $10 \mu \mathrm{mol} / \mathrm{L}$ dimethylenastron could significantly inhibit cell proliferation when cells were treated for $72 \mathrm{~h}$ (Figure 4B). These results thus demonstrate that the inhibitory 

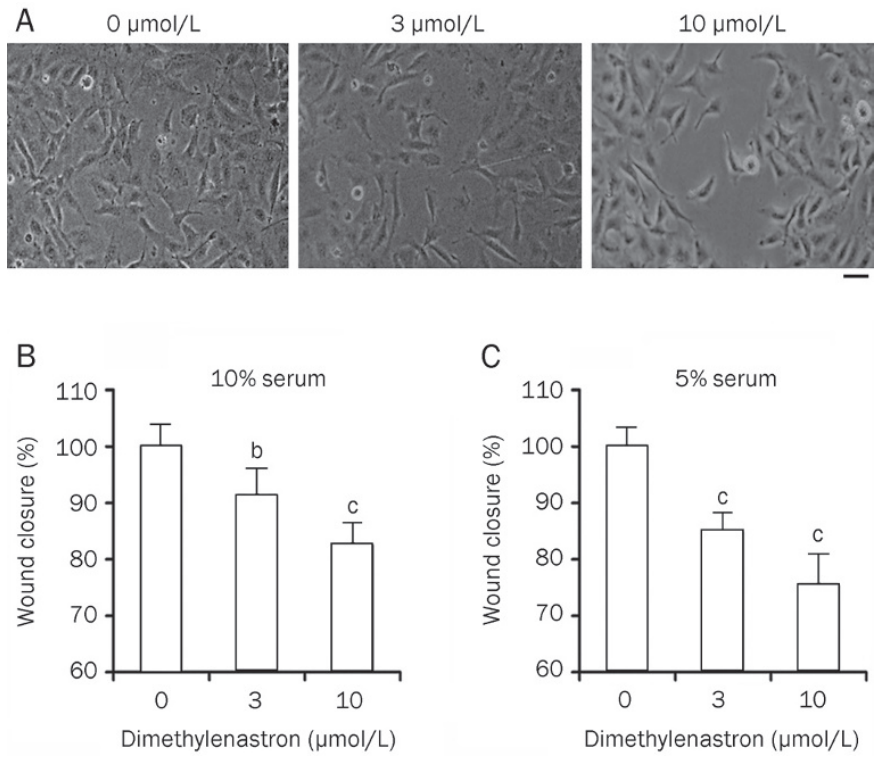

Figure 2. Determination of the effect of dimethylenastron on pancreatic cancer cell migration by wound healing assay. (A) PANC1 cells grown in serum-free medium were treated with 0,3 , or $10 \mu \mathrm{mol} / \mathrm{L}$ dimethylenastron and scratched. Culture medium containing $10 \%$ serum was added to allow for wound healing, and wound margins were imaged $24 \mathrm{~h}$ later. Scale bar, $50 \mu \mathrm{m}$. (B) Experiments were performed as in panel A, and the extent of wound closure was quantified by counting the number of migrating cells. (C) Experiments were performed as in A and B, except that culture medium containing $5 \%$ serum was used for wound healing. Mean \pm SD. $n=3 .{ }^{b} P<0.05,{ }^{c} P<0.01$ vs control.

effects of dimethylenastron on pancreatic cancer cell migration and invasion are independent of its effect on cell proliferation.

\section{Dimethylenastron allosterically inhibits the motor domain ATPase of Eg5}

To gain more mechanistic insight into how dimethylenastron inhibits Eg5 activity, we analyzed their interaction by molecular modeling. Dimethylenastron was docked onto the coordinates obtained from the crystal structure of $\mathrm{Eg} 5^{[18]}$, using standard DOCK methodology ${ }^{[19]}$, and the lowest-energy Eg5/ dimethylenastron interaction model was presented (Figure $5 \mathrm{~A})$. Detailed analysis revealed that dimethylenastron binds to the motor domain of Eg5 in a pocket close to the ATP/ADP binding pocket on Eg5; there exist a loop (E116-R119) and an a-helix (G110-F113) between the two binding pockets (Figure 5B). This model suggests that in the absence of ATP/ADP, dimethylenastron does not bind to Eg5 stably. Following ATP binding and hydrolysis to ADP, the ADP molecule pushes the aforementioned loop and a-helix to the dimethylenastron pocket and promotes the interaction between $\mathrm{Eg} 5$ and dimethylenstron.

Analysis of the ADP release rate has been useful for studying the effect of Eg5 inhibitors on its activity towards ATP hydrolysis ${ }^{[0]}$. To investigate whether dimethylenastron inhibits Eg5 activity by affecting ADP release, we analyzed the ADP release rate of Eg5 motor domain with the MANT-ADP
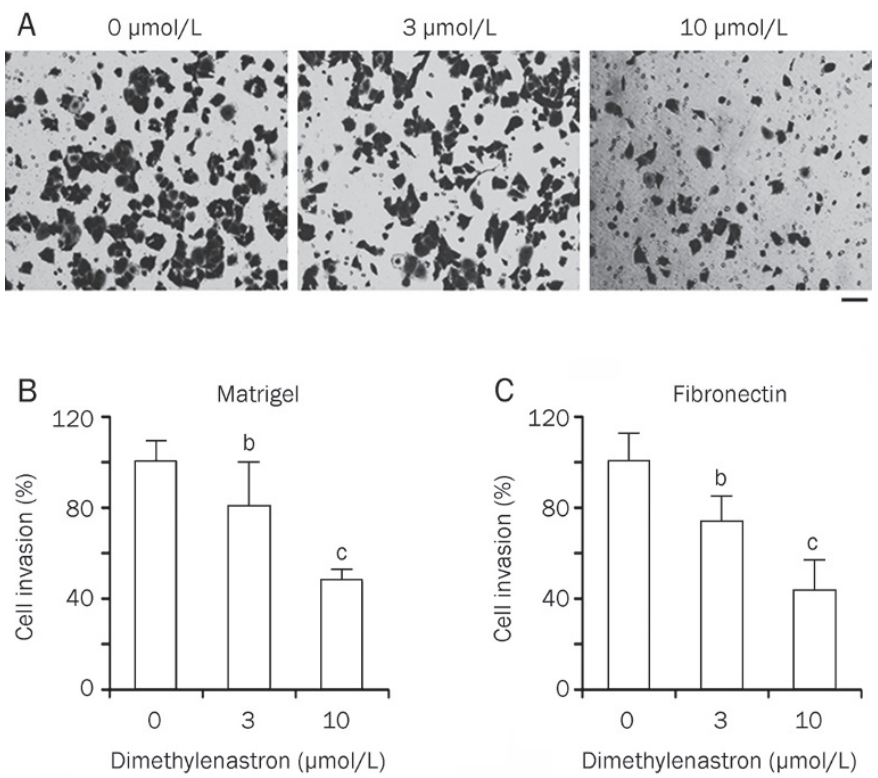

Figure 3. Dimethylenastron inhibits pancreatic cancer cell invasion as measured by transwell assay. (A) PANC1 cells were treated with 0,3 , or $10 \mu \mathrm{mol} / \mathrm{L}$ dimethylenastron and loaded to the transwell chambers (the upper surface of the transwell filters was coated with matrigel). After $24 \mathrm{~h}$, cells migrated to the underside of the transwell filters were stained with crystal violet solution and imaged. Scale bar, $50 \mu \mathrm{m}$. (B) Experiments were performed as in panel $A$, and the extent of cell invasion was determined by counting the number of invading cells. (C) Experiments were performed as in $A$ and $B$, except that the upper surface of the transwell filters was coated with fibronectin. Mean \pm SD. $n=3$. ${ }^{b} P<0.05$, ${ }^{\mathrm{c}} P<0.01$ vs control.

reagent, a fluorescent analog of ADP that displays increased fluorescence when bound to $\mathrm{Eg} 5^{[20]}$. As shown in Figure 5C, dimethylenastron significantly inhibited the basal rate of ADP release (in the absence of microtubules). In the presence of microtubules, dimethylenastron inhibited the rate of ADP release to a similar extent. These data thus suggest that dimethylenastron allosterically inhibits the motor domain ATPase of Eg5 by decreasing ADP release, independently of the binding of Eg5 to microtubules.

\section{Discussion}

The incidence of pancreatic cancer has been increasing at an alarming rate on a global scale over the past two decades. In spite of recent improvement in patient survival, the treatment of pancreatic cancer is still difficult. Our previous studies have shown that the Eg5 inhibitor dimenthylenastron inhibits the growth of pancreatic tumor both in vitro and in the mouse model $^{[11]}$. In the present study, our results show that dimethylenastron inhibits pancreatic cancer cell migration and invasion. These findings thus provide novel mechanistic insight into the anticancer effect of dimethylenastron, suggesting the potential of using this agent for pancreatic cancer treatment. Given that the ability to rapidly metastasize is the most deadly aspect of pancreatic cancer and that tumor metastasis results 

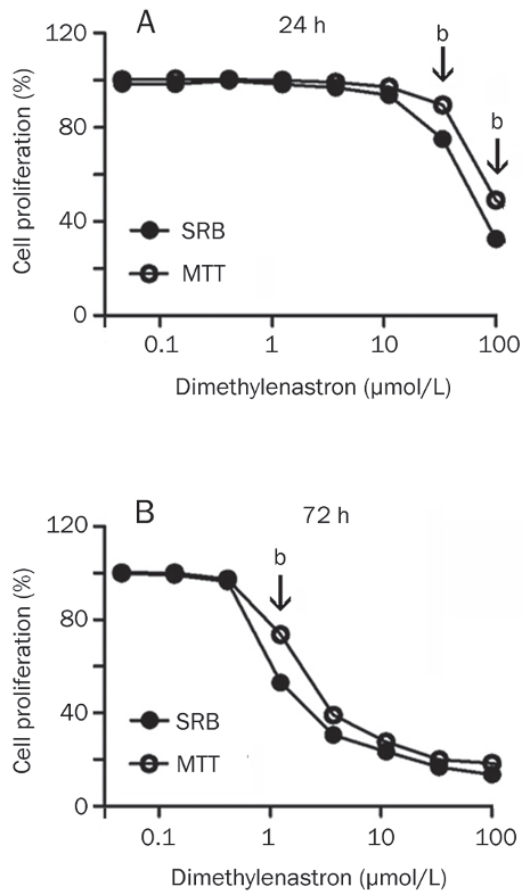

Figure 4. Dimethylenastron inhibits the proliferation of pancreatic cancer cells in a concentration-dependent manner. PANC1 cells were treated with gradient concentrations of dimethylenastron for $24 \mathrm{~h}(\mathrm{~A})$ or $72 \mathrm{~h}(\mathrm{~B})$. The percentage of cell proliferation was measured by sulforhodamine $B$ (SRB)- or MTT-based cell proliferation assays. ${ }^{\mathrm{b}} \mathrm{P}<0.05$ (SRB data versus MTT data).

mainly from cancer cell migration and invasion ${ }^{[14]}$, the use of dimethylenastron for pancreatic cancer management might decrease cancer metastasis and improve patient survival.

Our study has also demonstrated that the inhibitory effects of dimethylenastron on pancreatic cancer cell migration and invasion are independent of its effect on cell proliferation. At present, the molecular mechanism of how dimethylenastron decreases cancer cell motility remains elusive. It has been shown in a previous study that knockdown of myosin II in fibroblasts could enhance cell migration and that the increase in cell motility by knockdown of myosin II could be rescued by the inhibition of Eg5 activity ${ }^{[22]}$. Therefore, it would be important to investigate whether the inhibition of Eg5 activity in pancreatic cancer cells by dimethylenastron could trigger the myosin II-associated signaling pathway.

The effectiveness of most chemotherapeutic agents has been impaired by various side effects. In this study, by molecular modeling and analysis of ADP release rate, we find that dimethylenastron allosterically inhibits the motor domain ATPase of Eg5, independent of the Eg5/microtubule interaction. Although the relationship between the allosteric inhibition of Eg5 activity and the inhibition of migration and invasion of pancreatic cancer cells remains unclear, our data suggest that dimethylenastron might have few (if any) side effects as a chemotherapeutic agent, because it would not affect the normal physiological functions of Eg5 that require its interaction with
A

B
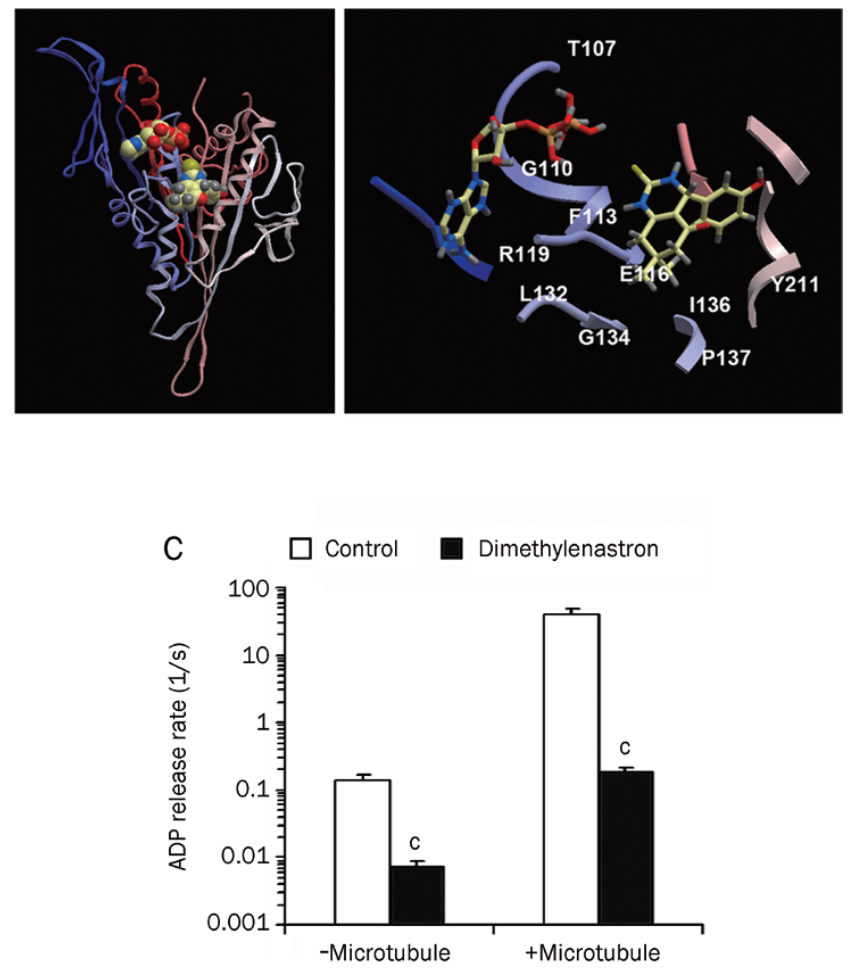

Figure 5. Dimethylenastron allosterically inhibits Eg5 activity by decreasing the rate of ADP release. (A) Schematic models showing the structure of Eg5 bound with ADP (upper) and dimethylenastron (lower). (B) Details of important interactions of ADP (left) and dimethylenastron (right) with the motor domain of Eg5, with red depicting oxygen, blue depicting nitrogen, yellow showing carbon, and grey representing hydrogen. Amino acid residues of Eg5 involved in the interaction with ADP and dimethylenastron are labeled. (C) Effects of dimethylenastron on the ADP release rate of Eg5 motor domain in the presence or absence of microtubules. Mean \pm SD. $n=3 .{ }^{c} P<0.01$ vs control.

microtubules.

Another important question is how Eg5 expression is regulated in normal cells and how it is highly expressed in pancreatic cancer cells. In our previous study, we presented evidence that cellular Eg5 level is down-regulated by parkin, an E3 ubiquitin ligase well known for its role in Parkinson's disease. Interestingly, parkin represses Eg5 gene transcription by Hsp70 monoubiquitination-dependent inactivation of the c-Jun $\mathrm{NH}_{2}$-terminal kinase pathway and blocking c-Jun binding to the activator protein 1 site in the Eg5 promoter, instead of triggering Eg5 protein degradation through the ubiquitinproteasome pathway ${ }^{[23]}$. In addition, the Bcr-Abl tyrosine kinase has been reported to regulate Eg5, although the molecular details remain unclear ${ }^{[6]}$. Considering that both parkin and Bcr-Abl have been implicated in tumorigenesis, it would be interesting to examine whether abnormalities in these proteins play a role in triggering Eg5 overexpression in pancreatic cancer cells. 


\section{Acknowledgements}

This work was supported by grants from the National Natural Science Foundation of China (31170820 and 90913021) and the Fok Ying Tung Education Foundation (111036).

\section{Author contribution}

Min LIU and Jun ZHOU designed research; Xiao-dong SUN, Xing-juan SHI, Xiao-ou SUN, Xiao-jing WU, Chang-fu YAO, and Hai-yang YU performed research; Deng-wen LI and Min LIU contributed new reagents or analytic tools; You-guang LUO and Jun ZHOU wrote the paper.

\section{References}

1 Valentine MT, Gilbert SP. To step or not to step? How biochemistry and mechanics influence processivity in Kinesin and Eg5. Curr Opin Cell Biol 2007; 19: 75-81.

2 Kapitein LC, Peterman EJ, Kwok BH, Kim JH, Kapoor TM, Schmidt CF. The bipolar mitotic kinesin Eg5 moves on both microtubules that it crosslinks. Nature 2005; 435: 114-8.

3 Castillo A, Morse HC 3rd, Godfrey VL, Naeem R, Justice MJ. Overexpression of Eg5 causes genomic instability and tumor formation in mice. Cancer Res 2007; 67: 10138-47.

4 Liu M, Wang X, Yang Y, Li D, Ren H, Zhu Q, et al. Ectopic expression of the microtubule-dependent motor protein Eg5 promotes pancreatic tumourigenesis. J Pathol 2010; 221: 221-8.

5 Hayashi N, Koller E, Fazli L, Gleave ME. Effects of Eg5 knockdown on human prostate cancer xenograft growth and chemosensitivity. Prostate 2008; 68: 1283-95.

6 Carter BZ, Mak DH, Shi Y, Schober WD, Wang RY, Konopleva M, et al. Regulation and targeting of Eg5, a mitotic motor protein in blast crisis CML: overcoming imatinib resistance. Cell Cycle 2006; 5: 2223-9.

7 Kapoor TM, Mayer TU, Coughlin ML, Mitchison TJ. Probing spindle assembly mechanisms with monastrol, a small molecule inhibitor of the mitotic kinesin, Eg5. J Cell Biol 2000; 150: 975-88.

8 Hotha S, Yarrow JC, Yang JG, Garrett S, Renduchintala KV, Mayer TU, et al. HR22C16: a potent small-molecule probe for the dynamics of cell division. Angew Chem Int Ed Engl 2003; 42: 2379-82.

9 Sakowicz R, Finer JT, Beraud C, Crompton A, Lewis E, Fritsch A, et al Antitumor activity of a kinesin inhibitor. Cancer Res 2004; 64: 327680.

10 Tao W, South VJ, Zhang Y, Davide JP, Farrell L, Kohl NE, et al. Induc- tion of apoptosis by an inhibitor of the mitotic kinesin KSP requires both activation of the spindle assembly checkpoint and mitotic slippage. Cancer Cell 2005; 8: 49-59.

11 Liu M, Yu H, Huo L, Liu J, Li M, Zhou J. Validating the mitotic kinesin Eg5 as a therapeutic target in pancreatic cancer cells and tumor xenografts using a specific inhibitor. Biochem Pharmacol 2008; 76 : 169-78.

12 Gartner M, Sunder-Plassmann N, Seiler J, Utz M, Vernos I, Surrey T, et al. Development and biological evaluation of potent and specific inhibitors of mitotic Kinesin Eg5. Chembiochem 2005; 6: 1173-7.

13 Jemal A, Siegel R, Ward E, Hao Y, Xu J, Thun MJ. Cancer statistics, 2009. CA Cancer J Clin 2009; 59: 225-49.

14 Ghaneh P, Costello E, Neoptolemos JP. Biology and management of pancreatic cancer. Gut 2007; 56: 1134-52.

15 Hezel AF, Kimmelman AC, Stanger BZ, Bardeesy N, Depinho RA. Genetics and biology of pancreatic ductal adenocarcinoma. Genes Dev 2006; 20: 1218-49.

16 Furukawa T, Duguid WP, Rosenberg L, Viallet J, Galloway DA, Tsao MS. Long-term culture and immortalization of epithelial cells from normal adult human pancreatic ducts transfected by the E6E7 gene of human papilloma virus 16. Am J Pathol 1996; 148: 1763-70.

17 Zhou J, Liu M, Aneja R, Chandra R, Lage H, Joshi HC. Reversal of P-glycoprotein-mediated multidrug resistance in cancer cells by the C-Jun $\mathrm{NH}_{2}$-terminal kinase. Cancer Res 2006; 66: 445-52.

18 Turner J, Anderson R, Guo J, Beraud C, Fletterick R, Sakowicz R. Crystal structure of the mitotic spindle kinesin Eg5 reveals a novel conformation of the neck-linker. J Biol Chem 2001; 276: 25496-502.

19 Kick EK, Roe DC, Skillman AG, Liu G, Ewing TJ, Sun Y, et al. Structurebased design and combinatorial chemistry yield low nanomolar inhibitors of cathepsin D. Chem Biol 1997; 4: 297-307.

20 Gilbert SP, Mackey AT. Kinetics: a tool to study molecular motors. Methods 2000; 22: 337-54.

21 Lieber M, Mazzetta J, Nelson-Rees W, Kaplan M, Todaro G. Establishment of a continuous tumor-cell line (panc-1) from a human carcinoma of the exocrine pancreas. Int J Cancer 1975; 15: 741-7.

22 Even-Ram S, Doyle AD, Conti MA, Matsumoto K, Adelstein RS, Yamada KM. Myosin IIA regulates cell motility and actomyosin-microtubule crosstalk. Nat Cell Biol 2007; 9: 299-309.

23 Liu M, Aneja R, Sun X, Xie S, Wang $\mathrm{H}$, Wu X, et al. Parkin regulates Eg5 expression by Hsp70 ubiquitination-dependent inactivation of c-Jun $\mathrm{NH}_{2}$-terminal kinase. J Biol Chem 2008; 283: 35783-8. 